\title{
artigo
}

Carvalho Silva, A.C.; Santos, F.M.S.; Gaudêncio, J.F.F.S.; Honorio, M.L.T.; Rodrigues da Silva, L.S.;

Morte materna relacionada a má/não assistência ao pré-natal

\section{Morte materna relacionada a má/não assistência ao pré-natal}

\author{
Maternal death related to poor/non-assistance of prenatal care \\ Muerte materna relacionada a mala/no atención al prenatal
}

\begin{abstract}
RESUMO
Introdução: A morte materna continua sendo uma problemática de saúde em inúmeras regiões do mundo, especialmente nas menos desenvolvidas. No ano de 2015, estimou-se que a razão de morte materna global foi de 216 por 100 mil nascidos vivos (NV), ocorrendo variações como de 542 por 100 mil NV na África a 16 por 100 mil NV na Europa. Objetivo: averiguar na literatura cientifica a morte materna relacionada a má/não assistência ao pré-natal. Método: Trata-se de um estudo bibliográfico, tipo revisão integrativa. Realizou-se a busca por artigos; com delimitação atemporal; nos idiomas português, inglês e espanhol; disponíveis na íntegra. Nas seguintes plataformas de dados: BDENF, LILACS, MEDLINE/BVS, SciELO e Science direct. Os dados foram organizados e apresentados em figuras e tabelas. Resultados: Dos 1563 estudos encontrados, 14 estava disponivel na BDENF, 60 na LILACS, 909 na MEDLINE/BVS, 0 na SciELO e 16561 na Science direct; contudo, após a leitura permaneceram apenas os que atendiam aos critérios para inclusão e exclusão descritos na metodologia, totalizando 4 estudos. Após a leitura dos estudos selecionados, os artigos foram categorizados em recortes temáticos, classificando o conhecimento produzido acerca do tema. Conclusão: A revisão integrativa elaborada possibilitou detectar os impactos da má/não assistência ao pré-natal na morte materna, e sua importância pra qualidade de atendimento prestado ao paciente.
\end{abstract}

DESCRITORES: Morte materna, Cuidado Pré-natal, Assistência em saúde.

\section{ABSTRACT}

Introduction: Maternal death remains a health issue in many regions of the world, especially in the least developed regions. In 2015, the global maternal death rate was estimated to be 216 per 100,000 live births (LB), with variations ranging from 542 per 100,000 LB in Africa to 16 per 100,000 LB in Europe. Objective: to investigate in the scientific literature maternal death related to poor/non-prenatal care. Method: This is a bibliographic study, type integrative review. The search for articles was carried out; with timeless delimitation; in Portuguese, English and Spanish; available in full. On the following data platforms: BDENF, LILACS, MEDLINE/BVS, SciELO and Science Direct. The data were organized and presented in figures and tables. Results: Of the 1563 studies found, 14 were available at BDENF, 60 at LILACS, 909 at MEDLINE/BVS, 0 at SciELO and 16561 at Science direct; however, after reading, only those who met the inclusion and exclusion criteria described in the methodology remained, totaling 4 studies. After reading the selected studies, the articles were categorized into thematic clippings, classifying the knowledge produced on the topic. Conclusion: The developed integrative review made it possible to detect the impacts of poor / non-prenatal care on maternal death, and its importance for the quality of care provided to the patient. DESCRIPTORS: Maternal Death, Prenatal Care, Delivery of Health Care.

\section{RESUMEN}

Introduction: Maternal death remains a health issue in many regions of the world, especially in the least developed regions. In 2015, the global maternal death rate was estimated to be 216 per 100,000 live births (LB), with variations ranging from 542 per 100,000 LB in Africa to 16 per 100,000 LB in Europe. Objective: to investigate in the scientific literature maternal death related to poor/non-prenatal care. Method: This is a bibliographic study, type integrative review. The search for articles was carried out; with timeless delimitation; in Portuguese, English and Spanish; available in full. On the following data platforms: BDENF, LILACS, MEDLINE/BVS, SciELO and Science Direct. The data were organized and presented in figures and tables. Results: Of the 1563 studies found, 14 were available at BDENF, 60 at LILACS, 909 at MEDLINE/BVS, 0 at SCiELO and 16561 at Science direct; however, after reading, only those who met the inclusion and exclusion criteria described in the methodology remained, totaling 4 studies. After reading the selected studies, the articles were categorized into thematic clippings, classifying the knowledge produced on the topic. Conclusion: The developed integrative review made it possible to detect the impacts of poor / non-prenatal care on maternal death, and its importance for the quality of care provided to the patient. DESCRIPTORES: Maternal Death, Prenatal Care, Delivery of Health Care.

RECEBIDO EM: 27/02/2021 APROVADO EM: 05/04/2021 


\section{Ana Carolina de Carvalho Silva}

Enfermeira (egressa) Faculdade da Escada (FAESC). Escada, Pernambuco (PE), Brasil.

ORCID: 0000-0003-0076-6412

\section{Fabiana Maria Sena Santos}

Enfermeira (egressa) Faculdade da Escada (FAESC). Escada, Pernambuco (PE), Brasil.

ORCID: 0000-0002-7384-9852

\section{Jessica Fernanda Freire da Silva Gaudêncio}

Enfermeira (egressa) Centro Universitário Maurício de Nassau (UNINASSAU). Recife, Pernambuco (PE), Brasil. ORCID: 0000-0003-3542-4658

\section{Maria Luciane Torres Honorio}

Enfermeira (egressa) Faculdade da Escada (FAESC). Escada, Pernambuco (PE), Brasil.

ORCID: 0000-0003-2110-0361

\section{Liniker Scolfild Rodrigues da Silva}

Sanitarista na modalidade residência pela Faculdade de Ciências Médicas (FCM)/Universidade de Pernambuco (UPE). Especialista em Enfermagem Obstetra na modalidade residência pela Faculdade de Enfermagem Nossa Senhora das Graças (FENSG)/ UPE. Recife, Pernambuco (PE), Brasil.

ORCID: 0000-0003-3710-851X

\section{INTRODUÇÃO}

A mortalidade materna, definida como a morte durante a gravidez ou no prazo de 42 dias após o final da gestação, é um problema de saúde pública global. Este é um indicador importante para analisar a saúde das mulheres, o desenvolvimento econômico e as desigualdades sociais em uma população. A morte materna continua sendo uma problemática de saúde em inúmeras regiões do mundo, especialmente nas menos desenvolvidas. No ano de 2015, estimou-se que a razão de morte materna global foi de 216 por 100 mil nascidos vivos (NV), ocorrendo variaçôes como de 542 por 100 mil NV na África a 16 por 100 mil NV na Europa ${ }^{(1,2)}$.

No Brasil, a redução da mortalidade materna é ainda vista com dificuldade para os serviços de saúde e para a sociedade em geral. As altas taxas identificadas caracterizam um grande problema de saúde pública, afetando de formas diferentes as regiões brasileiras, prevalecendo entre mulheres das classes sociais menos favorecidas. Se caracteriza ainda como uma grave violação dos direitos humanos das mulheres, uma vez que se trata de uma tragédia evitável em 92\% dos casos e ocorrerem em grande parte nos países em desenvolvimento ${ }^{(3)}$.

As mortes maternas e neonatais ocor- rem na maior parte ao longo da internação da mulher para o parto e nascimento; em torno de $15 \%$ das mortes são decorrentes de aborto inseguro; $51 \%$ das mortes neonatais ocorrem na primeira semana de vida e se relacionam majoritariamente com problemas decorrentes do parto; cerca de $70 \%$ das mortes das mulheres ocorrem por causas obstétricas diretas ${ }^{(4)}$.

Nos países subdesenvolvidos, as principais causas de morte materna são hemorragias pós-parto, distúrbios hipertensivos, sepse, partos obstruídos e complicações relacionadas ao aborto inseguro. Um dos maiores desafios para as diretrizes de desenvolvimento de políticas destinadas a reduzir a mortalidade materna é a sua real magnitude, mascarada por altos níveis de sub-registro de mortes e/ou subnotificação de causas de morte, especialmente em países em desenvolvimento, onde também acontecem cerca de três quartos de todos os nascimentos no planeta ${ }^{(5)}$.

Por isso, este estudo tem o objetivo de averiguar na literatura cientifica a morte materna relacionada a má/não assistência ao pré-natal.

\section{MÉTODO}

Trata-se de uma revisão integrativa percorridas com as seguintes etapas: elaboração da questão norteadora e objetivo do estudo; definição de critérios de inclusão e exclusão das produções científicas; busca de estudos científicos nas bases de dados e bibliotecas virtuais; análise e categorização das produções encontradas; resultados e discussão dos achados ${ }^{(6)}$.

Para o levantamento da questão norteadora, utilizou-se a estratégia PICo (P: Índice de Morte materna; I: Assistência ao pré-natal; Co: Assistência de qualidade). Desta forma, definiu-se a seguinte questão norteadora ${ }^{(7)} \mathrm{da}$ pesquisa: "Quais os impactos da má/não assistência ao pré-natal na morte materna?".

Para seleção dos artigos, utilizaram-se como critérios de inclusão: ser artigo original, disponíveis na integra, com delimitação atemporal, publicado em português, inglês ou espanhol, que respondessem ao objetivo do estudo e que possibilitasse o acesso pelo Virtual Private Network (VPN) da Universidade de São Paulo (USP). Foram excluídas as literaturas cinzas, bem como publicações repetidas de estudos em mais de uma base de dados os artigos que não responderam à questão norteadora do estudo. Justifica-se o estudo atemporal devido à população feminina sofrer com a falta ou deficiência de assistência ao pré-natal desde sempre.

O levantamento dos dados ocorreu durante o mês de janeiro e até o dia 14 de fevereiro de 2021 nas seguintes Bases de Dados: Base de dados de Enfermagem (BDENF); Literatura Latino-Americana e do Caribe em Ciências da Saúde (LILA- 


\section{artigo}

Carvalho Silva, A.C.; Santos, F.M.S.; Gaudêncio, J.F.F.S.; Honorio, M.L.T.; Rodrigues da Silva, L.S.;

Morte materna relacionada a má/não assistência ao pré-natal

CS); Medical Literature Analysis and Retrieval System Online via Biblioteca Virtual de Saúde (MEDLINE/BVS), Science Direct e na Biblioteca virtual Scientific Electronic Library Online (SciELO).

Buscaram-se os artigos indexados a partir dos Descritores em Ciências da Saúde (DeCS): "Morte Materna", "Cuidado Pré-natal", "Assistência em Saúde". Utilizaram-se os respectivos termos provenientes do Medical Subject Headings ( $\mathrm{MeSH}$ ): "Maternal Death", "Prenatal Care", "Delivery of Health Care”. A operacionalização e a estratégia de busca se deram a partir da combinação com operador booleano AND e OR, efetuando a busca conjunta e individualmente para que possíveis diferenças fossem corrigidas (Quadro 1).

A seleção dos estudos baseou-se no Preferred Reporting Items for Systematic Review and Meta-Analyse (PRISMA) $)^{(8)}$, com o objetivo de auxiliar no desenvolvimento de artigos. A princípio eliminaram-se por meio da leitura de títulos e resumos, estudos duplicados. Destes pré-selecionados, realizou-se leitura na íntegra, a fim de verificar os que atendem à questão norteadora e aos critérios de inclusão/exclusão. Construiu-se então a amostra final com estudos pertinentes aos critérios pré-estabelecidos (Figura 1).

Após a leitura dos artigos selecionados os estudos foram categorizados, classificando o conhecimento produzido em níveis de evidência de acordo com Melnyk e Fineout-Overholt ${ }^{(9)}$ : Nível I - Revisão sistemática, meta-análise ou diretrizes clínicas oriundas de revisões sistemáticas de ensaios clínicos randomizados e controlados; Nível II Ensaio clínico randomizado controlado; Nível III - Ensaios clínicos bem delineados sem randomização; Nível IV - Estudo de coorte e de caso-controle bem delineados;
Quadro 1. Estratégia de busca por base de dados, Recife, Pernambuco (PE), Brasil, 2021

\begin{tabular}{|c|c|c|}
\hline BASES DE DADOS & TERMOS DA BUSCA & RESULTADOS \\
\hline BDENF & Maternal death AND Prenatal Care AND & 14 \\
LILACS & Delivery of Health care & 60 \\
MEDLINE/BVS & Maternal death AND Prenatal Care OR & 16.561 \\
\hline Science Direct & Delivery of Health care & 0 \\
SciELO & & 17544 \\
\hline Total & \multicolumn{2}{|c|}{} \\
\hline Fonte: Elaboração própria.
\end{tabular}

Figura 1: Fluxograma do processo de seleção dos estudos primários adaptado do PRISMA, Recife, Pernambuco (PE), Brasil, 2021.

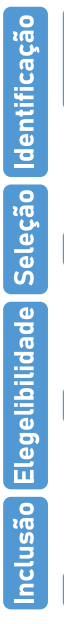

Publicações identificadas nas bases de dados $(n=17.544)$

BDENF - 14; LILACS - 60; MEDLINE/BVS - 90; Science Direct - 16561; ScIELO - 0

Excluídos por serem

duplicados $(n=16.500)$

Publicações rastreadas e elegiveis $(n=1.044)$

Excluídos após leitura de

títulos e resumos $(n=744)$

Estudos primários elegíveis para avaliação $(n=300)$

Excluídos por não

responderem à questão da

pesquisa $(\mathrm{n}=296)$

Estudos primários inclusos na amostra final $(n=4)$
Nível V - Revisão sistemática, de estudos descritivos e qualitativos; Nível VI - Estudo descritivo ou qualitativo; e por fim, Nível VI - Opinião de autoridades e/ou parecer de comissão de especialistas.

Obteve-se a sumarização das informações do corpus por meio de um instrumento: identificação do artigo original; autoria do artigo; ano de publicação; país; características metodológicas do estudo; e amostra do estudo. Analisaram-se os dados com o software Interface de R pour les Analyses Multidimensionnelles de Textes et de Questionnaires (IRAMUTEQ), versão 7.0, que possibilitou a análise da Classificação Hierárquica Descendente (CHD).

Objetivando uma melhor compreensão e visualização dos principais achados organizaram-se os dados apresentando-os em figuras e tabelas, expostos de forma descritiva.

\section{RESULTADOS}

Na tabela 1, os estudos levantados estão dispostos evidenciando seus títulos, autores, anos de publicação, delineamento, local e idioma. Onde se pode observar que a maioria dos estudos eram nacionais $(n=3)$, dois publicados em português e um em inglês, nos últimos 2 anos $(\mathrm{n}=3)$, e um artigo era internacional e publicado em inglês.

Após a leitura dos artigos selecionados, os estudos foram categorizados, classificando o conhecimento produzido sobre o tema, em níveis de evidência, majoritariamente Nível IV - Estudo de coorte e de caso-controle bem delineados. Os principais achados dispostos nos objetivos e conclusões, estão diretamente associados aos serviços de saúde, em especial, aqueles que prestam assistência ao pré-natal como exposto na tabela 2.

\section{DISCUSSÃO}

O Pré-Natal é imprescindível para que ocorra uma gestação, parto e pós-parto bem sucedidos. As pacientes precisam de um bom acompanhamento, onde avalia-se seu bem estar e do seu bebê, bem como alimentação, controle de peso e pressão arterial. Um pré-natal de qualidade é a chave para a redução da mortalidade materna em nosso 
Tabela 1: Resultados encontrados nos estudos de acordo com título, base de dados, autores, ano de publicação, delineamento, local e idioma. Recife, Pernambuco (PE), Brasil, 2021.

TÍTULO/BASE DE DADOS

Atrasos no atendimento obstétrico aumentam o risco de 1 eventos de quase-acidente de morbidade neonatal e morte: um estudo de caso-controle. / MEDLINE/BVS

2

Perfil sociodemográfico e assistencial da morte materna em Recife, 2006-2017: estudo descritivo. / LILACS

Pesquisa epidemiológica dos óbitos maternos e o cumprimento do quinto objetivo de desenvolvimento do milênio. / BDENF

A Associação de Cuidado Pré-natal Inadequado e Intensivo com Resultados Maternos, Fetais e Infantis: Um Estudo de Base Populacional em Manitoba, Canadá / Science Direct
AUTOR/ANO

Carvalho OMC, et al. (2020) ${ }^{(10)}$

Carvalho PI, et al. $(2020)^{(11)}$

Fernandes BB, et al. (2015) (12)

Heaman Ml, et al. (2019) ${ }^{(13)}$
DELINEAMENTO

LOCAL/IDIOMA controle

Brasil/ Inglês

Estudo qualitativo

Brasil / Português

Estudo epidemioló-

gico, retrospectivo e Brasil/ Português transversal

Estudo de coorte retrospectivo

Canadá / Inglês

Fonte: Elaboração própria.

Tabela 2: Principais resultados obtidos nos estudos de acordo com os níveis de evidências, objetivos e conclusões. Recife, Pernambuco (PE), Brasil, 2021.

\section{NÍVEL DE EVIDÊNCIA}

1

IV

2

VI

$3 \quad$ IV

$4 \quad$ IV

Fonte: Elaboração própria.
Avaliar a associação entre atrasos no atendimento obstétrico e eventos de quase mortalidade neonatal e óbito em maternidade pública de referência.

Descrever características sociodemográficas e assistenciais de mulheres que morreram por causa materna em Recife, Pernambuco, Brasil.

Identificar e descrever as características epidemiológicas dos óbitos maternos ocorridos entre 2000 a 2012 em um hospital de referência no estado de São Paulo, a fim de contribuir para a análise do cumprimento do quinto Objetivo de Desenvolvimento do Milênio.

Examinar a associação da utilização do cuidado pré-natal com os resultados maternos, fetais e infantis em Manitoba.

\section{CONCLUSÃO}

Os atrasos no atendimento obstétrico associados à presença de near miss e/ou óbito neonatal incluíram ausência ou inadequação do pré-natal, atraso no acesso aos serviços de saúde por falta de serviços especializados e conduta inadequada com a paciente.

As mortes ocorreram principalmente no puerpério e em negras; falhas assistenciais foram frequentes; é necessária melhor vigilância e acompanhamento dos serviços de saúde no período gravídico-puerperal, em Recife.

Há necessidade de investimento na assistência obstétrica para otimizar a redução das complicações durante a gestação e período puerperal, influenciando na redução da mortalidade materna.

Garantir que as mulheres recebam cuidados pré-natais adequados pode melhorar os resultados da gravidez. país. Também, o parto deve ocorrer em um ambiente seguro e realizado por uma equipe capacitada, buscando sempre o melhor tanto para a mãe quanto para o seu bebê, de forma que possa ter todo um suporte necessário em casos de anormalidades ${ }^{(14)}$.

Alguns estudos observaram que a maioria das mulheres realizam o pré-natal em unidades de Saúde da Família e algumas delas, são encaminhadas para o pré-natal de alto risco, contudo não são atendidas. Os municípios com alta abrangência na
Atenção Primária à Saúde proporcionam o pré-natal nas proximidades da residência, mesmo que a visita domiciliar por agentes de saúde e a aferição da pressão arterial não tenham sido observados em todas as mulheres. Problemas como estes, no pré-natal, dificultam o diagnóstico precoce e aumentam a chance de complicações na gravidez e no parto passem despercebidas. Inquérito nacional sobre parto e nascimento no Brasil identificou que, quando encaminhadas ao pré-natal de alto risco,
$11,5 \%$ das mulheres encontraram dificuldades para esse atendimento ${ }^{(11,15)}$.

A maior parte das mortes maternas são evitáveis, visto que existem soluções de cuidados de saúde para prevenir ou administrar complicações. É direito de toda mulher o acesso aos cuidados pré-natais durante a gestação, cuidados capacitados ao longo do parto e cuidados e apoio nas semanas após o parto. É fato que a saúde materna e do recém-nascido estão intimamente ligadas. No ano de 2015, estima-se que aproximada- 


\section{artigo}

Carvalho Silva, A.C.; Santos, F.M.S.; Gaudêncio, J.F.F.S.; Honorio, M.L.T.; Rodrigues da Silva, L.S.

Morte materna relacionada a má/não assistência ao pré-natal

mente 2,7 milhões de recém-nascidos morreram e outros 2,6 milhões de natimortos. É de suma importância que todos os partos sejam assistidos por profissionais de saúde qualificados, visto que o tratamento adequado pode fazer a diferença entre a vida e a morte da mãe e do bebê $\hat{e}^{(16)}$.

Uma limitação para o estudo foi o tamanho da amostra e a disponibilidade de artigos científicos para comparação dos resultados. Fazendo-se necessário a realização de mais estudos contendo uma amos- tra maior e possibilitando discussão acerca das implicações relacionadas aos impactos da má/não assistência ao pré-natal na morte materna.

\section{CONCLUSÃO}

A revisão integrativa elaborada possibilitou detectar os impactos da má/não assistência ao pré-natal na morte materna, e sua importância para a qualidade de atendimento prestado ao paciente. No entanto, há escassez de estudos que dão a verdadeira importância a implantação de uma assistência ao pré-natal de qualidade nos serviços de saúde, essenciais na prevenção, promoção da saúde, e educação permanente.

Com relação às implicações deste estudo para a prática assistencial, os resultados obtidos instigam a reflexões e discussões enquanto relevante aporte de referencial de conhecimento científico no campo da saúde e enfermagem, para o gerenciamento da qualidade do atendimento a paciente.

\section{REFERÊNCIAS}

1. Petersen EE, Davis NL, Goodman D, Cox S, Mayes N, Johnston E, et al. Vital signs: pregnancy-related deaths, United States, 2011-2015, and strategies for prevention, 13 States, 2013-2017. MMWR Morb Mortal Wkly Rep [Internet]. 2019 May [cited 2019 Oct 2];68(18):423-9. Available from: https://www.cdc.gov/mmwr/ volumes/68/wr/mm6818e1.htm

2. World Health Organization. United Nations Children's Fund. World Bank. United Nations. United Nations Population Division. Trends in maternal mortality: 1990 to 2015: estimates by WHO, UNICEF, UNFPA, World Bank Group and the United Nations Population Division [Internet]. Geneva: World Health Organization; 2015 [cited 2019 Apr 10]. 77 p. Available from: https:/apps.who.int/iris/bitstream/ handle/10665/194254/9789241565141_eng.pdf;jsessionid=90B5E6FF8F4F1E08DFD3D62AF9F07B62?sequence $=1$

3. Portal de Boas Práticas em Saúde da Mulher, da Criança e do Adolescente. Mortalidade Materna no Brasil - Boletim Epidemiológico n. ${ }^{\circ}$ 20/MS (Maio, 2020) [Internet] 2020 [cited 2021 feb 18]; Available from: https://portaldeboaspraticas.iff.fiocruz.br/atencao-mulher/mortalidade-materna-no-brasil-boletim-epidemiologico-n-o-20-ms-maio-2020/

4. Ruas CAM, Quadros JFC, Rocha JFD, Rocha FC, Andrade NGR, Piris ÁP et al. Perfil e distribuição espacial da mortalidade materna. Rev. Bras. Saude Mater. Infant. [Internet]. 2020 June [cited 2021 Feb 19]; 20(2):385-396. Available from: http://www.scielo.br/scielo.php?script=sci_arttext\&pid=S1519-38292020000200385\&lng=en. Epub Aug 05, 2020. http://dx.doi.org/10.1590/180693042020000200004.

5. Martins ACS, Silva LS. Epidemiological profile of maternal mortality. Rev Bras Enferm [Internet]. 2018;71(Suppl 1):677-83. [Thematic Issue: Contributions and challenges of nursing practices in collective health] http://doi.org/10.1590/0034-7167-2017-0624

6. Lisboa MT. Elements to formulate a research design. Mural Internacional, Rio de Janeiro, 2019; 10:e38439. https://doi. org/10.12957/rmi.2019.38439.

7. Araújo WCO. Recuperação da informação em saúde: construção, modelos e estratégias. ConCl: Conv. Cienc. Inform., 2020; 3(2):100-134.

8. Barbosa FT et al. Tutorial for performing systematic review and meta-analysis with interventional anesthesia studies. Brazilian
Journal of Anesthesiology (English Edition), 2019;69(3):299-306. https://doi.org/10.1016/j.bjan.2018.11.007

9. Melnyk BM, Fineout-OverholtE. Making the case for evidence-based practice. In B. M. Melnyk \& E. Fineout-Overholt. Evidence-based practice in nursing \& healthcare: a guide to best practice. 2005; 3-24. Philadelphia: Lippincot Williams \& Wilkins.

10. Carvalho OMC, Junior ABV, Augusto MCC et al. Delays in obstetric care increase the risk of neonatal near-miss morbidity events and death: a case-control study. BMC Pregnancy Childbirth, 2020; 20:437. https://doi.org/10.1186/s12884-020-03128-y

11. Carvalho PI, Frias PG, Lemos MLC, Frutuoso LALM, Figueirôa BQ, Pereira CCB et al. Perfil sociodemográfico e assistencial da morte materna em Recife, 2006-2017: estudo descritivo. Epidemiol. Serv. Saúde. 2020;29(1):e2019185. http://doi.org/10.5123/ s1679-49742020000100005

12. Fernandes BB, Nunes FBBF, Prudêncio PS, Mamede FV. Pesquisa epidemiológica dos óbitos maternos e o cumprimento do quinto objetivo de desenvolvimento do milênio. Rev. Gaúcha Enferm. 2015; 36 (spe): 192-199. http://doi.org/10.1590/1983-1447.2015.esp.56792

13. Heaman MI, Martens PJ, Brownell MD, Chartier MJ, Derksen AS, Helewa ME. The Association of Inadequate and Intensive Prenatal Care With Maternal, Fetal, and Infant Outcomes: a population-based study in manitoba, canada. Journal Of Obstetrics And Gynaecology Canada, 2019, 41 (7): 947-959, jul. http://doi.org/10.1016/j.jogc.2018.09.006.

14. Anchieta. Pré-natal pode reduzir o risco de mortalidade materna. [Internet] 2018 [cited 2021 feb 18] Available from: https:// www.hospitalanchieta.com.br/pre-natal-pode-reduzir-o-risco-de-mortalidade-materna/

15. Leal MC, Szwarcwald CL, Almeida PVB, Aquino EML, Barreto ML, Barros $F$, et al. Saúde reprodutiva, materna, neonatal e infantil nos 30 anos do Sistema Único de Saúde (SUS). Ciênc Saúde Coletiva [Internet]. 2018 jun [cited 2021 feb 18];23(6):1915-28. Available from: http:// www.scielo.br/pdf/csc/v23n6/1413-8123-csc-23-06-1915.pdf. http://doi.org/10.1590/1413-81232018236.03942018

16. OPAS. Folha informativa - Mortalidade materna. [Internet] 2018 [cited 2021 feb 18] Available from:https://www.paho.org/ bra/index.php?option=com_content\&view=article\&id=5741:folha-informativa-mortalidade-materna\&ltemid $=820$ 